\title{
Peroperative Tumoral Radial Dissemination During Cancellous Bone Graft Harvesting: Chondroma of the Finger
}

Sébastien $\mathbf{P}^{*}$

Department of plastic surgery, La Conception Hospital, France

*Corresponding author: Sébastien P, Department of plastic surgery, La Conception Hospital, 147 Boulevard Baille, 13005 Marseille, France, Tel: (33) (0) 688-716-694; E-mail: pascalsebastien@gmail.com

Received date: February 15, 2016; Accepted date: April 15, 2016; Published date: April 25, 2016

Copyright: @ 2016 Sébastien P. This is an open-access article distributed under the terms of the Creative Commons Attribution License, which permits unrestricted use, distribution, and reproduction in any medium, provided the original author and source are credited.

\begin{abstract}
Enchondromas are common bone tumors that preferentially affect fingers. It is essential to know that $10 \%$ of chondrosarcomas are secondary to preexisting lesions.

We present the case of a patient followed up for 30 years for a chondroma of a finger. Following increasing pain, curettage associated with radius bone graft was performed. However, the pain persisted postoperatively and inflammation and edema of the wrist and fingers appeared. Excisional biopsy was performed and the anatomopathologic results confirmed the sarcomatous transformation of the enchondroma and its dissemination to the radius. The oncological rules of surgery must be applied, even if the tumor appears to be benign in order to avoid dramatic consequences for the functional and vital prognosis of the patients.
\end{abstract}

Keywords: Chondrosarcomas; Sarcomatous; Carcinologic resection; Cancellous bone

\section{Introduction}

Enchondromas are common and benign bone tumors preferentially affecting fingers and especially phalanges (90\% of hand tumors) [1]. When they are symptomatic or voluminous, their treatment combines curettage and grafting [2]. It is essential to know that $10 \%$ of chondrosarcomas are secondary to preexisting lesions (enchondroma, exostosis) [3]. The oncological rules of surgery must be strictly applied even if the tumor appears to be benign. Otherwise, the consequences for the functional and vital prognosis of the patients can be dramatic. Numerous studies have noted the risk of tumor dissemination during invasive procedures such as biopsy or oncologic resection [3-5]. However, few studies have reported distant dissemination during a one-time reconstruction procedure. We describe the first case of a dramatic secondary transformation of a chondroma to a chondrosarcoma with dissemination to the radial harvesting site for reconstruction of a phalange.

\section{Case Report}

We describe the case of a 66-year-old woman, without medical history, followed up for 30 years for a bone lesion of the proximal phalange of the fourth finger of the right hand. A diagnosis of benign chondroma was made on radiographs without histological examination. In the absence of any symptoms, therapeutic abstention with annual radiological monitoring was decided.

Given her increasingly intense pain, surgery was performed in June 2012: resection by curettage associated with filling of the defect with ipsilateral distal radius cancellous bone graft and a bone substitute (Figure 1).

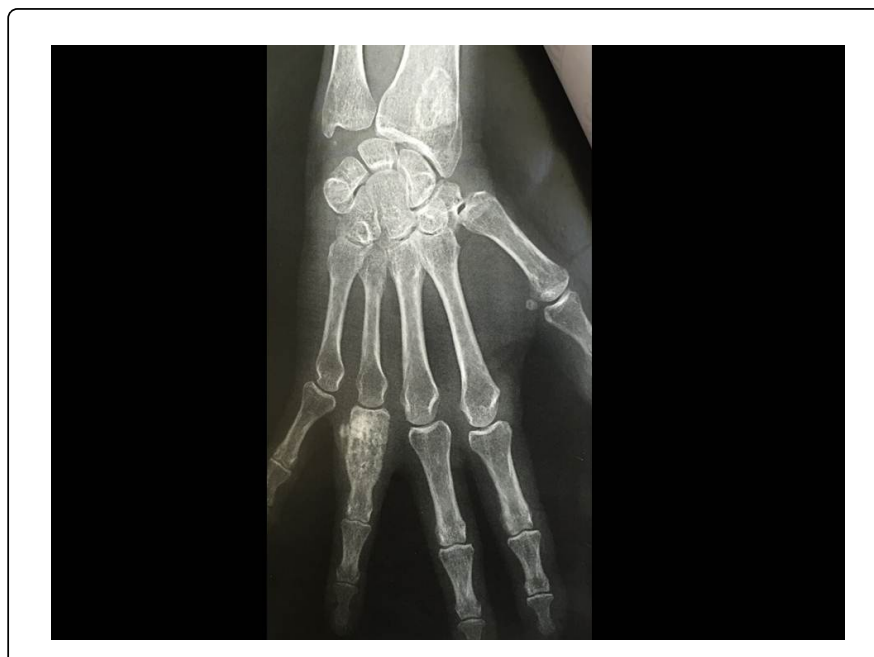

Figure 1: Postoperative radiographs of right wrist and hand. Cancellous bone graft and bone substitute at the phalange and bone substitute at the site of radial harvesting.

Histological analysis concluded an enchondroma without signs of malignancy that required simple monitoring. However, the pain persisted postoperatively and one year later, local inflammation and edema of the wrist and fingers appeared. The pain was constant and associated with hyperalgesic attacks. Imaging revealed a "mass syndrome" associated with phalange cortical lysis and soft tissue invasion. Osteolysis and cortical rupture was present on radial epiphysis (Figure 2). 


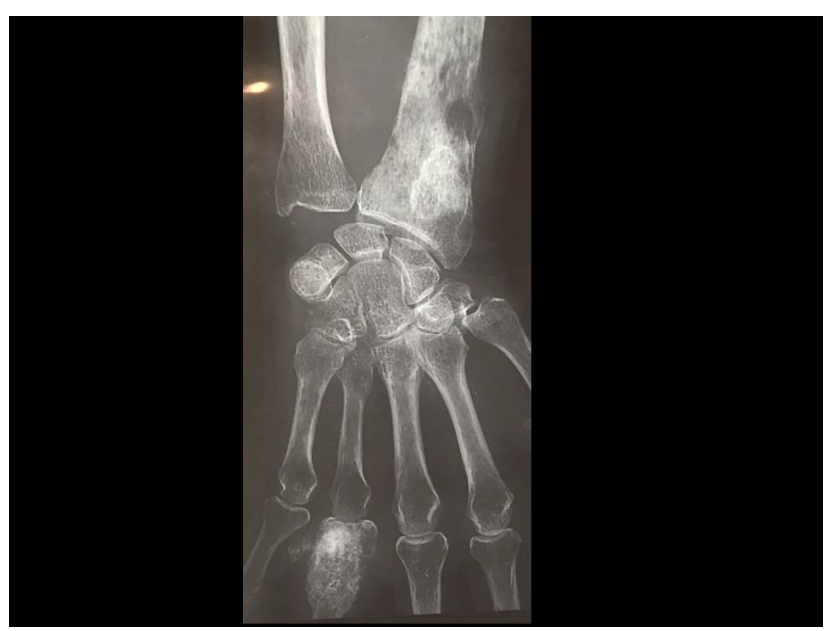

Figure 2: Radiographs of right wrist and hand at one year after the first surgery: "Mass syndrome" with irregular contours and cortical rupture of the proximal phalange associated with a peripheral reaction. Osteocondensation zone with osteolysis in relation to radial metaphysis.

Given the suspicion of secondary sarcomatous transformation and its preoperative dissemination at the radius, an exhaustive clinical and paraclinical work-up was performed including CT scan, MRI, scintigraphy and bone biopsies (finger and radius). The histology of the two sites showed discreet cytological atypias which could be part of a low-grade chondrosarcoma. Imaging supported this diagnosis (Figure 3).

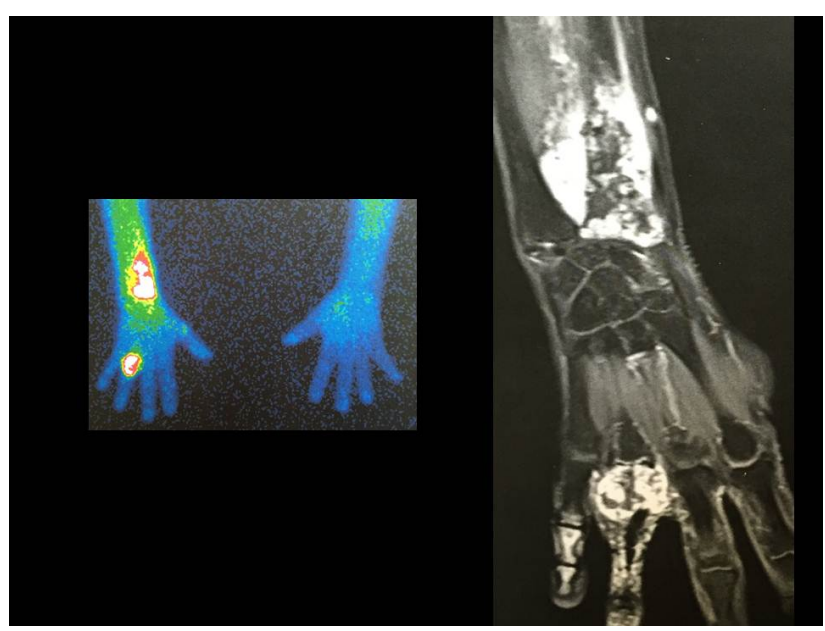

Figure 3: Scintigraphy (a) and MRI (b) one year after surgery. (a) intense hyperfixation areas at the fourth finger and distal radius. (b) osteolytic lesion of the proximal phalange of the fourth finger and the metaphysis and diaphysis of the radius associated with invasion of the soft tissue and cortical rupture: suggestive of a chondrosarcoma.

Broad excisional biopsy was performed at the radius and the phalange with the implementation of a cement spacer while waiting for the histopathological results. They confirmed the diagnosis: Grade 3 chondrosarcoma (WHO classification) in the phalange and dedifferentiated chondrosarcoma in the radius.

Following a multidisciplinary meeting and a negative tumor extension workup, we decided to perform amputation at the proximal quarter of the right forearm with histologically tumor-free margin. One month later, during postoperative monitoring, the patient presented pulmonary metastasis on the PET scan.

\section{Discussion}

The incidence of chondrosarcoma is low (fewer than 100 cases per year in France and 400 in the USA) [6], but its effects on survival have dire consequences (5\%-30\% survival at five years for a high degree of malignancy). It is essential to know that $10 \%$ of chondrosarcomas are secondary to preexisting lesions (chondroma, exostosis) [3]. Their treatment must be systematized with strict carcinologic rules [7]. In its simple form (asymptomatic, typical radiography), clinical and radiological monitoring is recommended. Surgical treatment by curettage and bone graft is performed in case of a large or symptomatic lesion. The age of a lesion does not necessarily mean that it is benign, so any atypical development of a lesion labeled "chondroma" should suggest the possibility of a secondary sarcomatous transformation to chondrosarcoma and require a multidisciplinary work-up in a reference center and an exhaustive medical and paramedical evaluation [8].

Biopsy (open or percutaneous) with anatomopathological examination is the gold standard for diagnosis of bone tumors [9]. However, one must remember that:

The risk of tumor dissemination with local metastasis is welldocumented in the literature because it could have severe consequences on survival and lead to therapeutic impasse or amputation. This has resulted in standardized techniques (the most direct approach in cooperation with the surgeon, tattooing of the point of entry in order to subsequently resect the puncture site, with care for the anatomical compartments) [10].

Sensitivity and specificity of the biopsy is not $100 \%$ [9]. It has been reported as being responsible for $18 \%$ of diagnostic errors. As described above, one must question false negatives that could present a tumor as incorrectly benign owing to unrepresentative cell architecture or sample size. Interpretation of pathological characteristics is difficult, particularly for chondrosarcomas because they often combine several degrees of malignancy within the same tumor. One solution to decrease this risk is to perform multiple histological samples guided by imaging to target the biopsy zone. The entire work-up must be performed in a reference center.

The risks associated with the biopsy dictate the strict observation of carcinologic rules, even if the tumor appears to be benign in order to decrease the risk of local or distant metastasis. We describe the first case of iatrogenic peroperative contamination of a chondrosarcoma on a cancellous bone graft harvesting site. In 2000, Ebelin described the peroperative spread of an osteosarcoma of the second metacarpal to an iliac crest during the harvesting of cancellous bone. The consequences were dramatic with the occurrence of pulmonary and vertebral metastasis leading to the patient's death in 3 years despite hemipelvis resection and complementary chemoradiotherapy [11]. In order to decrease iatrogenic peroperative metastasis, very strict carcinologic 
Citation: Sébastien P (2016) Peroperative Tumoral Radial Dissemination During Cancellous Bone Graft Harvesting: Chondroma of the Finger . J Cytol Histol 7: 407. doi:10.4172/2157-7099.1000407

Page 3 of 3

rules must be observed in cases of one-time reconstruction even when a benign tumor is suspected on biopsy:

2 distinct surgical stages,

2 separate surgical fields,

2 instrument tables with exclusive medical devices for each surgical stage,

Change of gloves for each stage,

If possible, preoperative evaluation of the residual bone defect assisted by imagery and primary harvesting.

Chondromas are common bone tumors but the possibility of a chondrosarcoma should preoccupy the surgeon. The oncological rules of surgery must therefore be strictly applied, even if a tumor initially appears to be benign given the possible dramatic consequences for the functional and vital prognosis of the patient.

\section{References}

1. Bauer RD, Lewis MM, Posner MA (1988) Treatment of enchondromas of the hand with allograft bone. J Hand Surg Am 13: 908-916.

2. Lin SY, Huang PJ, Huang HT, Chen CH, Cheng YM, et al. (2013) An alternative technique for the management of phalangeal enchondromas with pathologic fractures. J Hand Surg Am 38: 104-109.
3. Dubousset J, Forest M, Tomeno B (2001) Bone tumors. General diagnosis (biopsy and pathological anatomy). EMC - Appareil locomoteur 7: 1-8.

4. Zoccali C, Prencipe U, Erba F, Vidiri A, Di Filippo F (2009) Biopsy can determinate tumoral track contamination: A case report of chondrosarcoma. European Journal of Radiology Extra 7: 79-81.

5. Bacci G, Forni C, Longhi A, Ferrari S, Mercuri M, et al. (2007) Local recurrence and local control of non-metastati costeosarcoma of the extremities: a 27-year experience in a single. J Surg Oncol 96: 118-123.

6. Bergovec M, Kubat O, Smerdelj M, Seiwerth S, Bonevski A, et al. (2015) Epidemiology of musculoskeletal tumors in a national referral orthopedic department. A study of 3482 cases. Cancer Epidemiol 39: 298-302.

7. Sassoon AA, Fitz-Gibbon PD, Harmsen WS, Moran SL (2012) Enchondromas of the hand: factors affecting recurrence, healing, motion, and malignant transformation. J Hand Surg Am, 37: 1229-1234.

8. Bonvalot S, Vanel D, Terrier P, Le Pechoux C, Le Cesne A (2004) Principles for the treatment of soft tissue sarcoma in adults, EMC Techniques chirurgicales - Orthopédie-Traumatologie 2004: 1-14.

9. Preteselle O, Barral FG, Court L, Russlas B, Manet L (2003) Interest of percutaneous biopsy of bone lesions of tumor appearance J Radiol 84: 693-697.

10. Li ZF, Li JM, Yan J, ZYang P, Li X (2013)Prevention of contamination by biopsy needle track contamination using a novel adriamycinloaded gelatin sponge. World J Surg Oncol 11: 169.

11. Ebelin M, Missenard G, Nordin JY (2000) Iatrogenic tumor metastasis to the pelvis after treatment for hand osteosarcoma. A case report. Chir Main 19: 272-275. 